\title{
Performance Analysis and Evaluation of Hybrid Network using different Integrated Routing Protocols
}

\author{
Mr. Rajneesh Narula, \\ Assistant Professor, Adesh Institute of Engineering and Technology, Faridkot \\ raj_narula74@yahoo.com, \\ Mr. Kaushal \\ Research Scholar, Adesh Institute of Engineering and Technology, Faridkot \\ Kaushalgandhi786@gmail.com
}

\begin{abstract}
The increasing demand of computer communication networks are growing rapidly day by day. With the growing need to distribute applications across multiple networks and the availability of high capacity, high-performance intermediate switching nodes, and networks, an efficient routing mechanism has become the core requirement. This research primarily focuses on the design and performance of Hybrid Network incorporating different intra-domain routing algorithms. The performance evaluation of different routing algorithms for the transmission of video- and voice-data streams over Hybrid network is demonstrated in this work.. This allows multiple Ethernet point-to-point links to be bundled into one logical fullduplex channel for Fast Ethernet (10BaseT, 100BaseT, or 1000BaseX). These applications require some QoS support such as guaranteed bandwidth, less delay, less jittering effect and low error rate. The QoS relies on a number of factors along with a suite of robust routing protocols that help to accomplish the task of moving datagram from source to destination with high bandwidth and low delay rate. An effective intra-domain network routing protocol may make distributed applications more efficient across multiple networks with the availability of high capacity and high-performance. A variety of intra-domain routing protocols such as Routing Information Protocol (RIP) and Open Shortest First Protocol (OSPF), Interior Gateway Protocol (IGRP) and Enhanced Interior Gateway Protocol (EIGRP) are available and widely used in designing such high capacity and high performance networks with optimum QoS. We evaluate the performance of these intra-domain routing protocols with IS-IS to recommend the optimum routing protocol to use to provide optimum QoS by means of OPNET Simulator ${ }^{\mathrm{TM}}$. In this thesis work, the following objectives are considered and demonstrated.
\end{abstract}

\section{Council for Innovative Research}

Peer Review Research Publishing System

Journal: INTERNATIONAL JOURNAL OF COMPUTERS \& TECHNOLOGY

Vol 11, No.10

editor@cirworld.com

www.cirworld.com, member.cirworld.com 


\section{INTRODUCTION}

1.1 Background:-Today is the age of communication where internet is growing in leaps and bound. With the growth of Internet, the Internet Service Providers (ISPs) try to meet the increasing traffic demand by adopting new technology and improved utilization of existing resources. Routing of data packets can affect network utilization. Packets are sent along network paths from source to destination following a protocol. Routing is the process of moving data from one network to another network. Within a network, all hosts are directly accessible and do not need to pass data through a default gateway.

\subsection{ROUTING PROTOCOLS}

Routers are used to perform the routing process of forwarding packets between networks. Routers are connected to the edges of two or more networks to provide connectivity between them. These devices are usually dedicated machines with specialized hardware and software to speed up the routing process. These devices send and receive routing information to each other about networks that they can and cannot reach. Routers examine all routes to a destination, determine which routes have the best metric, and insert one or more routes into the IP routing table on the router. Computer communication networks are based on a technology that provides the technical infrastructure, where routing protocols are used to transmit packets across the Internet. The router has prior knowledge about the adjacent networks, which can assist in selecting the routes between two nodes. It is a protocol used to exchange routing information between autonomous systems. An EGP protocol allows knowing about all routes from its immediate neighbors on the internal network and the machines associated with them but lack of information about the rest of the Internet. Moreover, there is the Interior Gateway Protocol (IGP) that refers to the protocols used within a single autonomous system. An EGP determines whether a network is accessible from an autonomous system, while IGP will be responsible for dealing the routing within the autonomous system. Also, an autonomous system (AS) is a set of IP networks and IP devices that are managed by a single entity and have a common definition of Internet paths. To the outside world, an AS is a single entity that can be administered by one or more operators, while presenting a unified scheme for routing to the outside world [J. Hawkinson and T. Bates, 1996]. Figure 1.1 shows an example of a network of three AS, where each AS uses an IGP protocol to communicate. While EGP protocols are used to communicate with other AS.

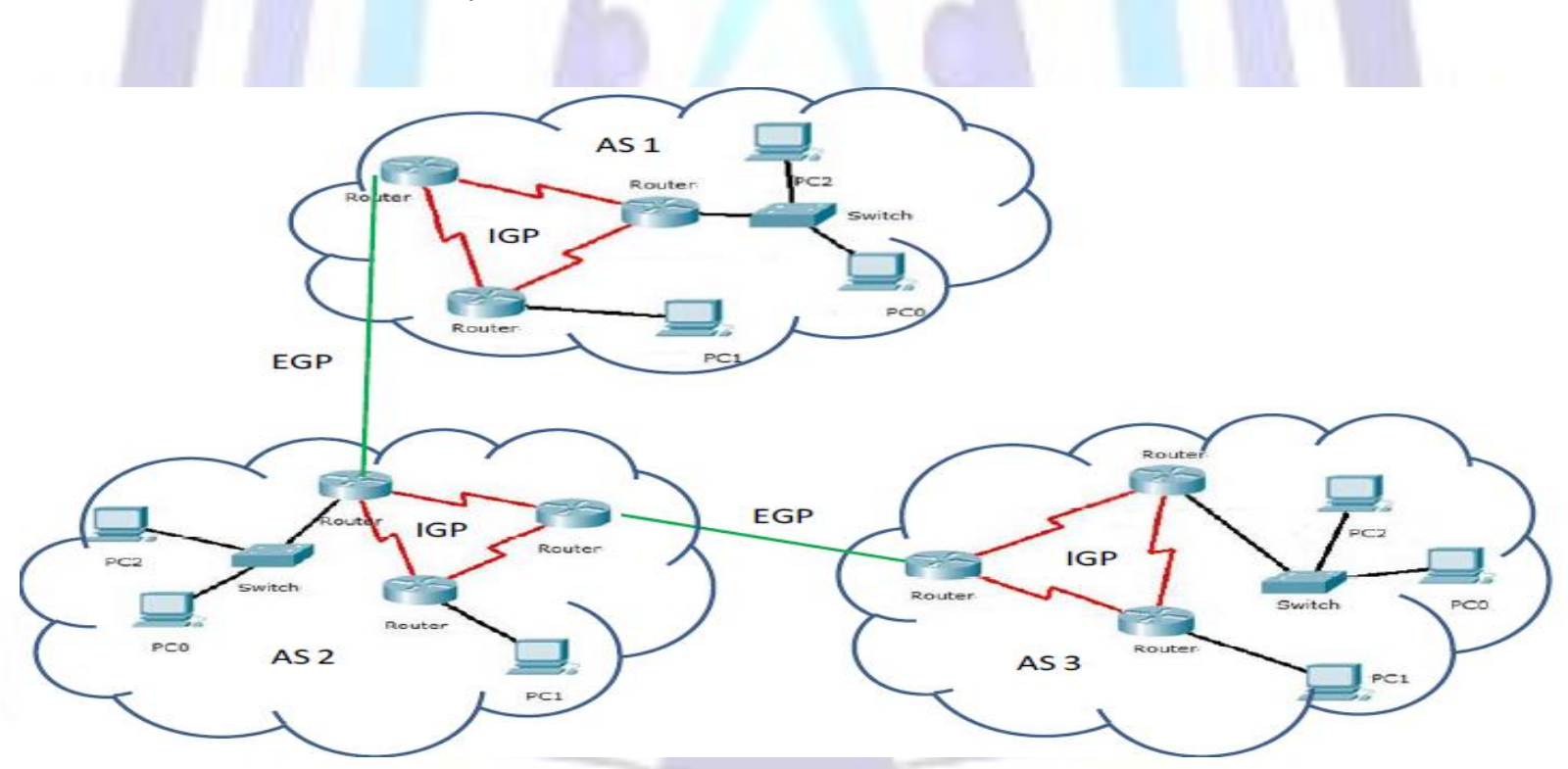

Figure 1.1 Example of network of three AS

A routing protocol is a communication scheme between several routers that can form a sub-network. A routing protocol allows the router to share connected network information with other routers as well as its proximity to other routers. The information obtained from another router, by the routing protocol, is used to create and maintain the routing tables. The routing table contains the known networks and the interfaces associated with such networks. The end user of a network does not know how data flows between the devices.

\subsection{Classification of Routing Protocols}

The classifications of routing protocols are

1. Static and dynamic routing protocols.

2. Distance Vector and Link State routing protocols.

3. Classful and Classless routing protocols. 
1.3.1 Static and dynamic routing protocols:-Static routing is a routing process whose routing table follows a manual construction and fixed routes at boot time. The routing table needs to be updated by the network administrator when a new network is added and discarded in the AS. Static routing is mainly used for small networks. Its performance degrades when the network topology is changed routing. It usually provides more control for the system administrator in order to maintain the whole network. In static routing, the network has more control over the network. On the contrary, dynamic routing is a routing protocol in which the routing tables are formed automatically such that the neighboring routers exchange messages with each other. The best route procedure is conducted based on bandwidth, link cost, hop number and delay [Kisten and S.Ping-Tsai Chung, 2003]. The protocol usually updates these values. Dynamic routing protocol has the advantage of shorter time spent by the administrator in maintaining and configuring routes. However it has diversity problems like routing loops and route inconsistency.

\subsubsection{Distance Vector and Link State routing protocols}

\section{Distance vector routing protocol:}

Distance vector routing protocol presents routes as a function of distance and direction vectors where the distance is represented as hop count metrics and direction is represented as exit interface. In DVR, the Bellman Ford algorithm is used for the path calculation where the nodes take the position of the vertices and the links. In DVR, for each destination, a specific distance vector is maintained for all the nodes used in the network.

Functionality of Routing: - Distance vector routing protocol uses the Bellman Ford algorithm for identifying the best path. For calculating the best network path, different methods are used by the Distance Vector (DV) routing protocols. But, for all DV routing protocols, the main characteristic of such algorithms is found to be same. For identifying the best path in a network, various route metrics are used to calculate the direction and the distance. For example, EIGRP uses the diffusion update algorithm (DUAL) for calculating the cost which is needed to reach a destination. Routing Information Protocol (RIP) uses hop count for choosing the best path and IGRP determines the best path by taking information of delay and bandwidth availability.

\section{Link State Routing:}

Link State Routing (LSR) protocols are also known as Shortest Path First (SPF) protocol where the function of each router is to determine the shortest path among the network. Each router maintains a database called link state database. It is mainly used to describe the topology of the AS. The Link State Advertisements (LSA) is responsible for exchanging the routing information among the nodes. The information of the neighbours is found in each LSA of a node and any change in link information of a neighbour's node is communicated through LSAs by flooding.

Two of the most common types of LSR protocols are OSPF and IS-IS. OSPF determines the shortest distance between nodes based on the weight of the link.

\subsection{Classful and Classless routing protocols}

\section{Based on the subnet mask, routing protocols are divided into Classful and Classless routing} as below:

1. Classful Routing - In Classful routing, subnet masks perform the same functionality all through the network topology and this kind of protocol does not send information of the subnet mask. If a router calculates a route, it will perform the following functions:-

1. Routers use the same subnet mask which is directly connected to the interface of the major network.

2. When the router is not directly connected to the interface of the same major network, it applies Classful subnet mask to the route.

\section{Classless Routing}

In classless routing, the subnet mask can be changed in network topology and routing updates are included. Most networks do not depend on classes for being allocated these days and also for determining the subnet mask, the value of the first octet is not used. Classless routing protocols.

RIP is an intra-domain routing protocol used inside an autonomous system. It is a very simple protocol based on distance vector routing. IGRP is a distance routing protocol invented by Cisco. Routing protocols find a path between network nodes; if multiple paths exist for a given node then the shortest path is selected by protocol. Each protocol has a cost metric that it applies to each path. The path with lowest metric is selected by protocol [2]. Metrics to compare one routing protocol with another are based on End to End delay (in sec), delay variation (in sec), point-to-point throughput (in bits/sec), point-to-point queuing delay (in seconds), traffic received (in bits/sec), and IP processing delay (in seconds) have been computed. In this paper we have integrated ISIS routing Protocol with RIP and OSPF and calculated metrics. 


\section{RELATED WORK}

Many routing protocols have been proposed to for but it can still be addressed that integration of IS-IS with RIP and OSPF to enhance the performance for real-time application have never been made. For the most part, previous studies of different routing protocols such as EIGRP, OSPF and RIP have been done based on simulation [5], in which the authors have concentrated on comparative performance and in detailed simulation study carried out in the IP network. In this paper, in terms of selecting the right protocol, comparison and evaluation of the routing protocols have been done by integrating an IS-IS/RIP and IS-IS/OSPF based on performance metrics such as network convergence, CPU utilization, throughput, queuing delay, and bandwidth utilization. However, our work has broadly concentrated on the comparative performance analysis of the different routing behaviors by integrating IS-IS with RIP and OSPF for the real-time applications (e.g. voice conferencing and video streaming).

\section{METHODOLOGY}

We divide our work into two parts. First of all, we design a network model with RIP-ISIS, simulate it by using OPNET and observe the impact of using this hybrid network alone for real time applications. Secondly, in the same network scenario, we implement OSPF with ISIS and simulate the network model to observe the impact of using OSPF-ISIS alone for real time applications. We compare and evaluate performance of these hybrid networks protocols based on quantitative metrics such as convergence duration, packet delay variation, end-to-end delay, packet loss and throughput. We employ Optimized Network Engineering Tool (OPNET) as a simulator to evaluate and to analyze the comparative performance of these routing protocols. Third part we integrated IS-IS with RIP and evaluate performance of integrated IS-IS/RIP to measure convergence duration, packet to packet delay variation, end-to-end delay, packet loss, throughput, Utilization. Lastly we created scenario for integrated IS-IS/OSPF and measure its performance for the same metrics. We compared the integrated IS-IS/RIP IS-IS/OSPF to find whether integrated model increases performance.

\section{RESULTS}

\subsection{Simulation Setup and Working}

Simulation can be defined to show the eventual real behaviour of the selected system model .It is used for performance optimization on the basis of creating a model of the system in order to gain insight into their functioning. We can predict the estimation and assumption of the real system by using simulation results.

In this thesis, network simulator, Optimized Network Engineering Tools (OPNET) modeller 9.1 has been used as a simulation environment. OPNET is a simulator built on top of discrete event system (DES) [OPNET IT Guru University LAB Manuals] and it simulates the system behaviour by modelling each event in the system and processes it through user defined processes [OPNET Document, hppt://www.opnet.com/documentations.html]. OPNET is very powerful software to simulate heterogeneous network with various protocols. Many pre-defined types of nodes are present and almost all widely used protocols and technologies are supported. The supported operating system is Windows only. The source code for protocols implementation is available; the application source code is closed. The application is quite sophisticated; it provides many settings for almost everything and it may be difficult for the beginner to get into it Therefore, it is intended rather for the networking professionals than students.

\subsection{Structure of the OPNET}

OPNET is a high level user interface that is built as of $C$ and $C++$ source code with huge library of OPNET function [Simulation and Tools for Telecommunication, OPNET07.pdf].

1. Network domain: Physical connection, interconnection and configuration can be included in the network model. In figure 3.1, it represents over all system such as network, sub-network on the geographical map to be simulated.

\subsection{Simulation Study}

The protocols used in this thesis are IS-IS integrated with RIP and IS-IS integrated with OSPF IS-IS integrated with EIGRP routing protocol. The proposed routing protocols are compared and evaluated based on some quantitative metrics such as End to End delay, Delay variation, point-to-point queuing delay, IP processing delay, point-to-point utilization, point-topoint throughput and network traffic. These protocols are intended to use to get better performance of one over the other for real time traffic such as video streaming and voice conferencing in the entire network.

\subsection{Designing Steps}

1. Create Network Model.

2. Choose Statistics.

3. Run Simulation.

4. Analysis Results. 


\subsection{Network Topology}

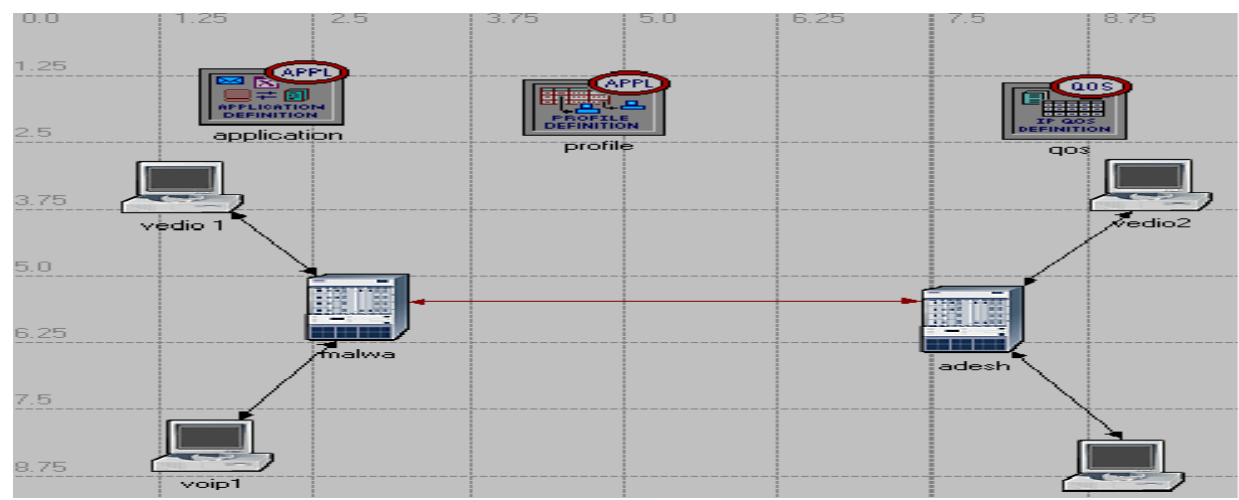

Figure 4.5: Network Topology

In this thesis, Two scenarios are created that consists of four Ethernet workstation connected with routers are configured by using RIP, OSPF,IS-IS integrated with RIP and IS-IS integrated with OSPF routing protocols. The network topology shown in figure 4.5 is composed of the following network devices and configuration utilities:

\section{CS_7000 Cisco Routers}

2. PPP_DS3 Duplex Link

3. PPP_DS1 Duplex Link

4. Ethernet Workstation

5. Application Configuration

6. Profile Configuration

7. QoS Attribute Configuration

An Application Definition Object and a Profile Definition Object named correspondingly Application Configuration and Profile Configuration in the figure 4.5 is added from the object palette into the workspace. The Application Configuration allows generating different types of application traffic. As far as real time applications are concerned in this, the Application Definition Object is set to support Video Streaming (Light) and Voice Conferencing (PCM Quality). A Profile Definition Object defines the profiles within the defined application traffic of the Application Definition Objects. In the Profile Configuration, two profiles are created. One of the Profiles has the application support of Video Streaming (Light) and another one has Voice Conferencing (PCM Quality) support. One Video Server is connected to Router Malawi that is set to the Video Streaming under the supported services of the Video Server. Quality of Service (QoS) plays an important role to provide better quality and to guarantee a minimum amount of bandwidth during congestion in the network. In order to implement QoS, IP QoS Object called QoS Parameters taken into the workspace where it has been used to enable and deploy First In First Out (FIFO). Two VolP workstations are connected to each other by routers which are set to the VolP under the supported services of the Voice workstations. Quality of Service (QoS) plays an important role to provide better quality and to guarantee a minimum amount of bandwidth during congestion in the network. In order to implement QoS, IP QoS Object called QoS Parameters taken into the workspace where it has been used to enable and deploy first in first out (FIFO).

\subsubsection{IS-IS RIP Scenario}

In this figure 4.6, RIP routing protocol is enabled first for all routers on the network. After configuring routing protocols, individual DES statistics was chosen to select performance metrics and to measure the behaviour of this routing protocol. Then simulation run time was set to 15 minutes. 


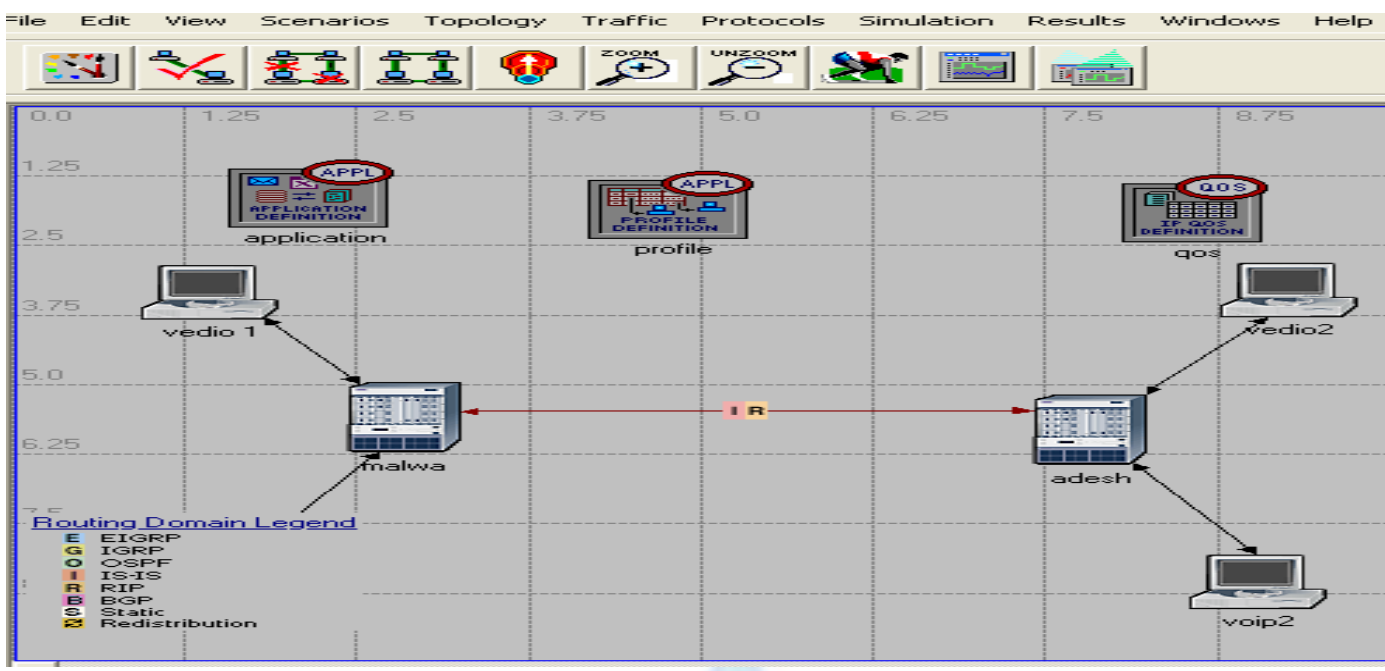

Figure 4.6: Network Topology With IS-IS RIP Enabled

\subsubsection{IS-IS OSPF Scenario}

Figure 4.7 show that first task is to set routing configuration of hybrid network IS-IS with OSPF as a routing protocol for this network topology. Then individual DES statistics was chosen that would be viewed in the results from the DES menu. Finally, time duration to run the simulation was set to 15 minutes.

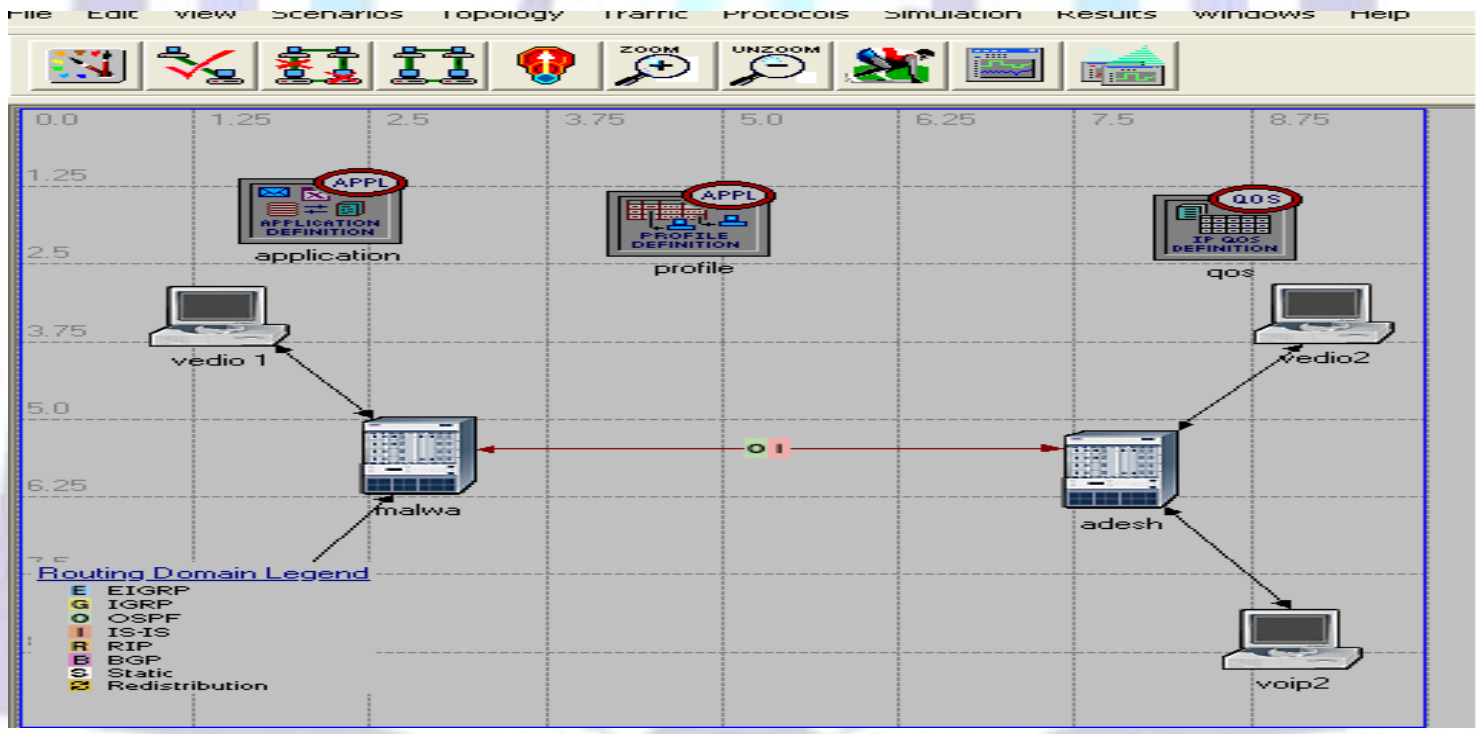

Figure 4.7: Network Topology with IS-IS OSPF Enabled

\subsection{Measurements}

In this section, measurements of the performance metrics such as network traffic, IP processing delay, utilization, point-topoint throughput and point-to-point queuing delay, End to End delay, Delay variation for video and voice transmission are done from the acquired results of Discrete Event Simulation

\subsection{Simulation Results and Analysis}

In this section, we present a comparative analysis of RIP and OSPF. There are two network models, which are configured and run as $1^{\text {st }}$ scenario with RIP alone, $2^{\text {nd }}$ one with OSPF alone. The links that have been used in these scenarios are PPP_DS1 and PPP_DS3. The data rate for PPP_DS1 and PPP_DS3 links are 1.544 Mbps and 44.736 Mbps, respectively. We consider background utilization in connecting the workstations to router, the link utilization and analyze the variation of each defined parameters. Link utilization is set in increasing order from normal $0 \%$ to $100 \%$. Table 4.1 shows the corresponding link utilization 


\begin{tabular}{|c|c|c|c|}
\hline Time in (sec) & $\begin{array}{c}\text { Link utilization in } \\
(\%)\end{array}$ & $\begin{array}{c}\text { Link value in (bps) } \\
\text { For PPP_DS1 }\end{array}$ & $\begin{array}{c}\text { Link value in (bps) } \\
\text { For PPP_DS3 }\end{array}$ \\
\hline 0 & 0 & 0 & $13420800\left(44736000^{\star} 0.3\right)$ \\
\hline 300 & 30 & $763200\left(1544000^{*} 0.3\right)$ & $22368000\left(44736000^{*} 0.5\right)$ \\
\hline 500 & 50 & $1080800\left(1544000^{*} 0.7\right)$ & $31315200\left(44736000^{\star} 0.7\right)$ \\
\hline 700 & 70 & $1389600\left(1544000^{\star} 0.9\right)$ & $40262400\left(44736000^{*} 0.9\right)$ \\
\hline 900 & 90 & $1544000\left(1544000^{\star} 1\right)$ & $44736000\left(44736000^{*} 1\right)$ \\
\hline 1000 & 100 & & \\
\hline
\end{tabular}

.Table 4.1 Link utilization values

OPNET is configured to acquire the graphical results of various network elements, which include video and voice traffic, router and links. In this section, graphed results for defined quantitative parameters are presented. Simulation duration of each scenario lasts for 900 seconds. Video and voice traffic starts at 90 seconds.

\subsubsection{End to End delay}
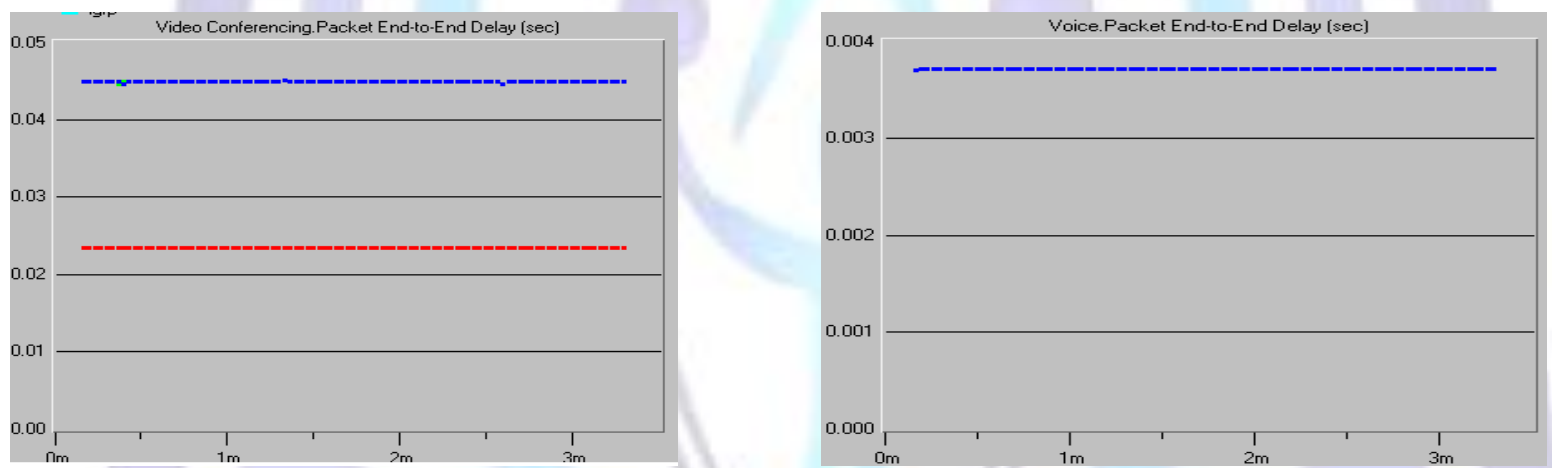

Figure 4.12: point to point queuing Delay different Routing protocols for transmission

Figure 4.13: point to point queuing Delay using using different Routing protocols for video voice transmission

Figures 4.12 shows that in view of point to point queuing delay for the video data streams, the hybrid network performs better when used with ISIS RIP routing protocols as compare to IS-IS OSPF enabled network which implies that the data packets have to wait for a longer average waiting time at routers that increase the probability of drop of data packets in case of ISIS OSPF enabled networks. In contrast Figure shows that in view of point to point queuing delay for the voice data streams, the hybrid network performs better when used with ISIS OSPF routing protocols as compare to IS-IS RIP enabled network which implies that the data packets have to wait for a longer average waiting time at routers that increase the probability of drop of data packets in case of integrated ISIS with RIP enabled networks. Further, the graphs shows that the point to point queuing delay is more for video data stream as compare to VolP traffic. 


\subsubsection{IP Processing Delay}

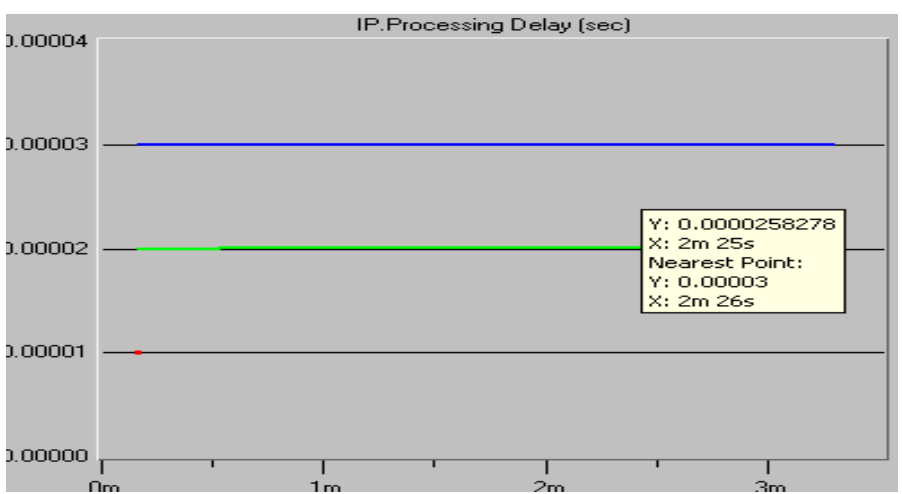

Figure 4.14: IP Processing Delay in sec using different Routing protocols

The IP processing delay for our network also evaluated as low by incorporating Isls-OSPF routing protocols as compare to IS-IS RIP enabled network as shown in Figure

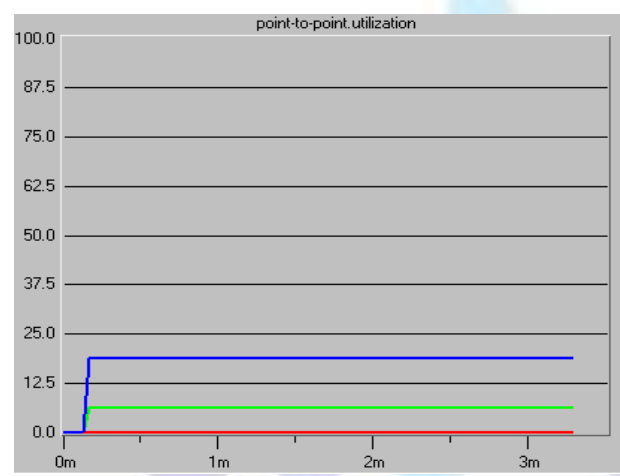

Figure 4.15: point to point utilization using different Routing protocols for Video transmission

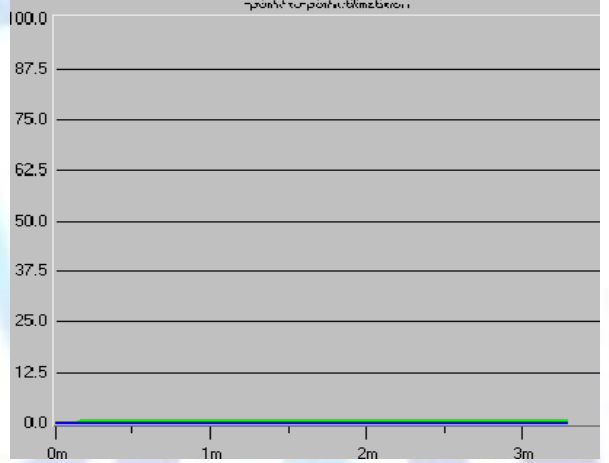

Figure 4.16: point to point utilization using different Routing protocols for voice

Furthermore, Figures 4.15 shows that the point to point utilization, a parameter related to the use of the channel disregard of throughput, of our simulative network is more for ISIS-OSPF than ISIS-RIP. Figure 4.16 shows that our simulative network is same with IS-IS RIP and OSPF routing protocols. Utilization parameter counts not only with the data bits but also with the transmission overhead, consists of preamble sequences, frame headers and acknowledge packets, which make use of the channel. Otherwise, the throughput would not be only associated to the efficiency of the protocol but also to retransmissions resultant from quality of the channel. In a simplistic approach, channel efficiency can be equal to channel utilization.

\subsubsection{Point to Point Throughput}

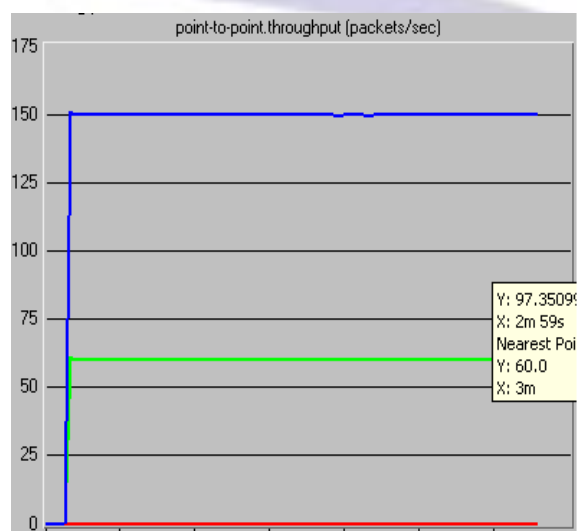

Figure 4.17: point to point throughput using different Routing protocols for video. for voice.

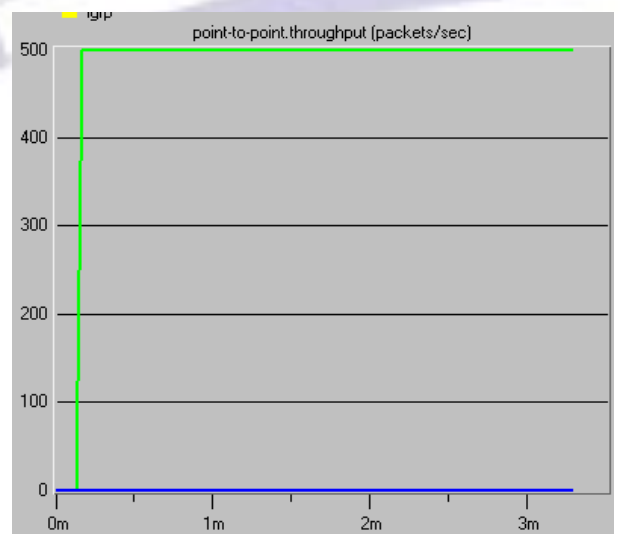

Figure 4.18: point to point throughput using different Routing protocols 
The point to point throughput, a key parameter to determine the rate at which total data packets are successfully delivered and received through the channel in the network, of our simulative network is Higher with ISIS- OSPF than ISIS-RIP routing protocols in case of video-data stream as depicted in Figure 4.17. In contrast, throughput in voice-data stream, of our simulative network is Same in cases of voice-data stream as depicted in Figure 4.18.

\subsubsection{Traffic Received:}

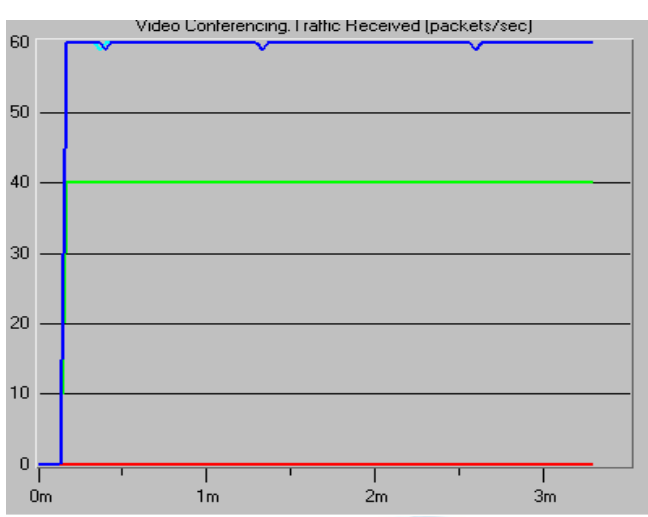

Figure 4.19: Traffic Received using different protocols for Video transmission

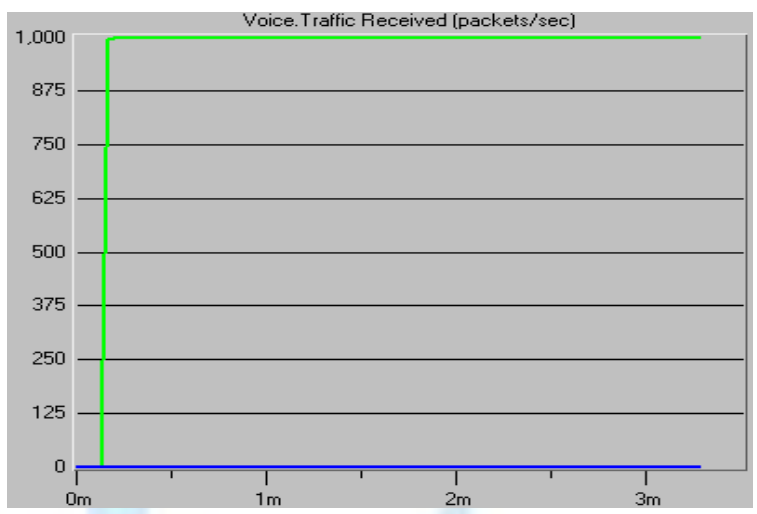

Figure 4.20: Traffic Received using different Routing Routing protocols for voice transmission

Furthermore, Figures 4.19 shows that in view of traffic received for the video data streams, the hybrid network ISIS OSPF performs better than ISIS-RIP whereas in case of voice-data stream Figure 4.20 shows our simulative network performs better when used with IS-IS RIP routing protocols as compare to IS-IS OSPF enabled network which implies that the traffic generated by IS-IS OSPF is doubled than to IS-IS RIP. These scenarios are the related work and we have calculated these parameters so as to compare with our own integrated scenarios and analyze the results against previous work to compare and find which one is better. The results been calculated and analyze in tabular form.

\section{CONCLUSION \& FUTURE WORK}

\subsection{Conclusion}

Interior routing protocols like RIP, OSPF, IGRP and EIGRP are widely being used in computer networking. In this thesis, we have reported a comparative analysis of Two routing protocols such as integrated IS-IS with RIP and ISIS with OSPF. In this section, the comparative analysis has been done in the same network with IS-IS RIP against ISIS OSPF routing protocols for real time applications. Performance has been evaluated on the basis of some parameters aimed to figure out the effects of routing protocols. For comparing these protocols performances, we have presented a Table:

\begin{tabular}{|l|c|c|}
\hline \multicolumn{1}{|c|}{ Parameters } & Best in Video & Best in Voice \\
\hline End to End Delay & ISIS RIP & ISIS RIP \\
\hline Delay Variation & ISIS OSPF & ISIS OSPF \\
\hline Queuing Delay & ISIS OSPF & ISIS OSPF \\
\hline IP Processing Delay & ISIS RIP & ISIS OSPF \\
\hline Utilization & ISIS-OSPF & ISIS RIP \\
\hline Throughput & ISIS-OSPF & ISIS OSPF \\
\hline Traffic Received & ISIS OSPF & \\
\hline
\end{tabular}

Table 5.1: Comparison between IS-IS RIP and IS-IS OSPF on various performance parameters for video \& voice data transmission

In view of this, we come across that the IS-IS RIP routing protocol performs better compared to ISIS OSPF in terms of End to End Delay, IP Processing Delay. ISIS OSPF has less queuing delay, and more throughput in case of video-data stream transmission with the available bandwidth. In contrast, voice data stream shows that IS-IS RIP routing protocol performs better as compared to IS-IS OSPF in view of End to End Delay, Queuing delay and Utilization, where as IS-IS OSPF routing protocol is better as compared to IS-IS RIP in terms of Delay Variation and IP Processing Delay. It remains same in terms of Utilization and traffic received with the available bandwidth but by considering other important network parameters, calculated in this 
work, used to evaluate the efficiency of a network, we can conclude that OSPF is the more efficient routing protocol to be used to deploy in small to medium sized network.

\subsection{Future Work}

The following areas still need to be explored to further optimize the algorithm and make it suitable for all types of application categories:

In the future, a research work will be done on the explicit features of all the routing protocols can be implemented in real time for more realistic results. IPv6 would be a predominant in future, these protocols can be tested in IPv6 version as well for an efficient working and the security issues of these protocols can be taken as future work.

It also recommends if anyone interested in research they can combine all the routing protocols with IS-IS, and make one advanced routing protocol. This can be done by analyzing the source code of each protocol and make a modification on the codes.

\section{REFERENCES}

[1]. Peter Martini, Otto Spaniol, "File transfer in high-speed networks” IEEE journal,vol-6, pp-987-990, july 1998.

[2]. Pun, H. "Convergence Behavior of RIP and OSPF Network Protocols". High-Performance Communication Networks, Morgan Kaufmann Publisher, pp:163-165, 1998.

[3]. Young-Bae Ko and Nitin H. Vaidya, "Location-Aided Routing in mobile and Ad-hoc networks" National Science on wireless networks, pp. 307-321, June 2000.

[4]. Sejun Song and BaekYoung Choi," Emerging Technologies and Factory Automation”, Vol 1, pp 315-323 , oct 2001.

[5]. Nohl, A.R, Molnar, G "The convergence of the OSPF routing protocol" (Ericsson Res., Ericsson Hungary Ltd., Budapest, Hungary); . Source: Periodica Polytechnic Electrical Engineering, v 47, n 1-2, p 89-100, 2002 WDM optical networks Concepts, Design and Algorithms, Prentice Hall of India Pvt. Limited, 2002.

[6]. Schneider, M. and T. Nemeth, "A simulation study of the OSPF-OMP routing algorithm". Computer Networks, Vol. 39 (4), pp: 457-468,2002.

[7]. Min Ying-Hua, "Survey on Computer Routing",CJC, vol No.6, pp: 641-649,2003.

[8]. Reza Azizi and Gurcu Oz, "Resource allocation and performance analysis problems in optical networks," Report, Helsinki University of Technology Networking Laboratory, 2004.

[9]. Korkmaz, T. and M. Krunz," OSPF-based hybrid approach for scalable dissemination of QoS parameters", Computer Networks, Vol. 46(2), pp: 273-293, 2004.

[10]. Peter Kok Keong Loh, Hsu Wen Jing, and Yi Pan," Reliable and efficient communications in sensor networks", Journal of Parallel and Distributed Computing, vol 67, pp 922-934, August 2007.

[11]. Dr Chandra Shekar Reddy Putta," Performance of ad-hoc network routing protocols in IEEE 802.11",ICCCT, pp. 371376,2010.

[12]. Talal Mohamed Jaffar, "Simulation based Routing Protocols Analysis ",Aug 2007.

[13]. Jinhua Guo, "Reinforce Network Theory with OPNET Simulation", journal of Information Technology Education, vol-6, 2007.

[14]. Ahmed Mahmoud, " OSPF traffic Routing Protocol in Hybrid Network", International Conference on Network and Parallel Computing Workshop, pp 676-682,sep 2007.

[15]. Franck Le," Understanding Routing Redistribution”, International conference, Beijing, China,2008.

[16]. Alex A. Stewart and Marta F. Antoszkiewicz, “Route Analysis and Management System”, 2009.

[17]. Wendwossen Anjelo and Esuendale Lemma, "Performance Comparison of EIGRP/IS-IS and OSPF/IS-IS", Nov-2009.

[18]. Mohammad Nazrul Islam and Md. A. Ullah, " Simulation-Based Comparative Study of EIGRP and OSPF for Real Time Applications", 2010.

[19]. Dong (Don) Xu, "Performance Analysis of EIGRP, OSPF, and RIP Based on OPNET", IJCA, vol-26,2011.

[20]. M. Shaad Zolpirani, M.-M. Bidmeshki, S. G. Miremadi "The Effect of Routing-Update Time on Networks Performability", pp655-658, 2007.

[21]. Talal Mohamed Jaffar, "Simulation-Based Routing Protocols Analysis (Thesis)" Ph.D.

Dept. Elect. Eng., Georgia Institute of Technology, 2007.

[22]. Ruhani Ab Rahman, Murizah Kassim, "Performance analysis of routing protocol in WiMAX network”,ICSE,2011.

[23]. Al-Saud, K.A. Tahir, H.M.; El-Zoghabi, A.A.Saleh, "Performance evaluation of 
secured versus non-secured EIGRP routing protocol" (Dept. of CS \& Eng., Qatar Univ.,

Doha, Qatar); Source: Proceedings of the 2008 International Conference on Security \& Management (SAM 2008), p 292-7, 2008

[24]. Xianhui Che ; Lee J. Cobley 'VoIP Performance over Different Interior Gateway Protocols' Journal: International Journal of Communication Networks and Information Security Year: 2009 Vol: 1 Issue: 1

[25]. Dr Chandra Shekar Reddy Putta," Performance of ad-hoc network routing protocols in IEEE 802.11”,ICCCT, pp. 371376,2010 .

[26].Hamidreza Mahini; Reza Berangi; Alireza Mahini 'MLET: A Power Efficient Approach for TCAM Based, IP Lookup Engines in Internet Routers', Journal: International journal of Computer Networks \& Communications, 2010 Vol: 2

[27]. Sapna , Manju Sharma, Harpreet Kaur 'Performance Evaluation of Hybrid network Using RIP \& IGRP for Different Applications' Published in 7th International Symposium on High-capacity Optical Networks and Enabling Technologies, Honet ( 2010) held at Egypt.

[28]. Thorenoor, S.G."Dynamic Routing Protocol Implementation Decision between EIGRP, OSPF and RIP Based on Technical Background Using OPNET Modeler" WiproTechnol.,Bangalore, India) Source: Proceedings of the 2010 Second International Conference on Computer and Network Technology (ICCNT 2010), p 191-5, 2010

[29]. Vasudha Arora, C.RamaKrishna, "Performance of three routing protocols AODV, DSR and WRP for FTP, TELNET and CBR traffic",IJCSR,vol-21,2012. 